\title{
Behavior management approach for agitated behavior in Japanese patients with dementia: a pilot study
}

This article was published in the following Dove Press journal:

Neuropsychiatric Disease and Treatment

20 December 2012

Number of times this article has been viewed

\author{
Junko Sato' \\ Shutaro Nakaaki \\ Katsuyoshi Torii' \\ Mizuki Oka² \\ Atsushi Negi' \\ Hiroshi Tatsumi ${ }^{3}$ \\ Jin Narumoto 4 \\ Toshi A Furukawa ${ }^{5}$ \\ Masaru Mimura ${ }^{2}$ \\ 'Department of Psychiatry and \\ Cognitive-Behavioral Medicine, \\ Nagoya City University Graduate \\ School of Medical Sciences, Nagoya, \\ ${ }^{2}$ Department of Neuropsychiatry, Keio \\ University School of Medicine, Tokyo, \\ ${ }^{3}$ Department of Health Science, \\ Faculty of Psychological and Physical \\ Science, Aichi Gakuin University, \\ Nagoya, ${ }^{4}$ Department of Psychiatry, \\ Graduate School of Medical Science, \\ Kyoto Prefectural University \\ of Medicine, Kyoto, ${ }^{5}$ Department \\ of Health Promotion and Human \\ Behavior (Cognitive-Behavioral \\ Medicine), Kyoto University Graduate \\ School of Medicine/School of Public \\ Health, Kyoto, Japan
}

Correspondence: Shutaro Nakaak Department of Neuropsychiatry, Keio University School of Medicine, 35 Shinanomachi, Shinjuku-ku, Tokyo 160-8582, Japan

Tel +8I3 53633829

$\mathrm{Fax}+81353790187$

Email hzi05510@nifty.com
Background: Agitated behaviors are frequently observed in patients with dementia and can cause severe distress to caregivers. However, little evidence of the efficacy of nonpharmacological interventions for agitated behaviors exists for patients with dementia. The present pilot study aimed to evaluate a behavioral management program developed by the Seattle Protocols for patients with agitated behaviors in Japan.

Methods: Eighteen patients with dementia (Alzheimer's disease, $n=14$; dementia with Lewy bodies, $n=4$ ) participated in an open study testing the effectiveness of a behavioral management program. The intervention consisted of 20 sessions over the course of 3 months. The primary outcomes were severity of agitation in dementia, as measured using the Agitated Behavior in Dementia scale (ABID) and the Cohen-Mansfield Agitation Inventory (CMAI).

Results: The behavioral management program resulted in significant reductions in total scores on both the ABID and CMAI. Although both physically agitated and verbally agitated behavior scores on the ABID improved significantly, symptoms of psychosis did not improve after the intervention.

Conclusion: The behavioral management technique may be beneficial to distressed caregivers of patients with dementia. In the future, a well designed study to develop the behavioral management program more fully is needed.

Keywords: Alzheimer's disease, dementia with Lewy bodies, agitated behavior, behavioral management, Agitated Behavior in Dementia scale

\section{Introduction}

Agitated behaviors are common in patients with Alzheimer's disease, dementia with Lewy bodies, and frontotemporal dementia. ${ }^{1}$ The prevalence of agitation increases with the severity of dementia, ${ }^{2}$ resulting in serious daily functional impairments because agitated behaviors are complex phenomena affected by interactions between cognitive impairment, pain, mental discomfort, and environmental factors, including the need for social contact and overstimulation. ${ }^{3}$ Agitated behaviors are problematic because they can cause severe distress to the caregivers of patients with dementia.

Antipsychotic medications have long been used to treat agitated behaviors in patients with dementia. Although atypical antipsychotic medications are known to be effective, ${ }^{4}$ the US Food and Drug Administration issued a warning in 2005 indicating that the pharmacological treatment of behavioral problems in patients with dementia was associated with an increased mortality risk. Several recent meta-analyses have agreed that serious adverse effects, such as cerebrovascular events, may occur in patients with dementia who receive antipsychotic medications..$^{5,6}$ Thus, nonpharmacological 
interventions are needed to manage agitated behaviors in this group of patients.

A number of nonpharmacological interventions have been devised. However, little evidence of the efficacy of these interventions for neuropsychiatric symptoms is available. A few placebo-controlled, randomized clinical trials of behavioral management techniques have demonstrated significant effects in reducing behavior problems in patients with dementia. ${ }^{7-9}$ These trials have been conducted as the Seattle Protocols and were developed by researchers in Seattle, WA. Several recent reviews ${ }^{10-12}$ have indicated that while other randomized clinical trials for nonpharmacological interventions have resulted in clinical meaningful improvements, insufficient research is available to generalize the efficacy of nonpharmacological interventions for behavioral problems in patients with dementia. In terms of some non-dementia-specific psychological tasks, interventions focused on direct-care nursing tasks associated with activities of daily living ${ }^{13}$ did not produce significant reduction in any disruptive behaviors. Suhr et al ${ }^{14}$ observed significant reductions in behavioral symptoms after the use of behavioral management techniques, including progressive muscle relaxation. However, this randomized clinical study had a small sample $(n=17)$. It is important that not only family members, but also professional staff in nursing and residential homes, receive sufficient education and training in communication techniques and behavioral management for dementia patients. Recent reviews ${ }^{12,15}$ have shown that several studies with structured staff education programs have yielded high-quality randomized clinical trial designs.

Some studies ${ }^{16,17}$ have had well trained staff educate family members either by performing family visits or making telephone contact. Other studies ${ }^{18,19}$ have directly trained health professionals, such as assisted living residence or care staff in nursing or residential homes, to develop skills in behavioral management. Although these studies resulted in significant clinical improvements in some aspects of either patient or caregiver outcome (eg, depression, perceived changes in well-being, burden), they failed to observe a significant reduction in behavioral problems, such as agitated behaviors, in dementia patients. Together, these findings suggest a lack of evidence supporting a decrease in behavioral problems after behavioral management interventions in patients with dementia.

Except for case reports, ${ }^{20}$ the use of behavioral management techniques for patients with dementia has not yet been established in Japan. Here we report a 12-week, open-label, prospective, pilot study which aimed to examine the effectiveness of a behavioral management program for patients with dementia and agitated behaviors.

\section{Materials and methods Participants}

The baseline sample consisted of Japanese patients with Alzheimer's disease or dementia with Lewy bodies who attended the outpatient clinic of Yagoto Hospital between December 2010 and March 2012. The diagnostic evaluation included a complete history and physical examination, routine blood tests (including evaluation of serum vitamin B12 levels and thyroid function), either a magnetic resonance imaging or computed tomography scan of the brain, and neuropsychological testing. Inclusion criteria were a diagnosis of probable Alzheimer's disease according to National Institute of Neurological and Communication Disorders and Stroke/Alzheimer Disease and Related Disorders Association criteria $^{21}$ or a diagnosis of probable dementia with Lewy bodies according to consensus guidelines, ${ }^{22}$ either the absence of antipsychotic medications or a stable dose of antipsychotic medications during the intervention period, and at least a 2-week history of two or three agitated behaviors occurring at least once weekly.

Patients were excluded if other neurological diseases were present, there was a previous history of mental illness or substance abuse before the onset of dementia, either an magnetic resonance imaging or a computed tomography scan had revealed focal brain lesions, the patient's Mini-Mental State Examination ${ }^{23}$ score was less than 11, or reliable informed consent could not be obtained from the patient and/ or relatives. The study protocol was approved by the ethics committee of Yagoto Hospital. Both the subjects and the caregivers were informed of the purpose of this study and its procedures, and were asked to sign a consent form.

\section{Clinical assessments}

Before the start and after the completion of the intervention, the following tests were conducted to assess cognitive and behavioral problems. Two interview-based questionnaires and one self-reported questionnaire were administered to all the caregivers, and one questionnaire was used to assess the patients with dementia. A well trained neuropsychologist who was blinded to the intervention administered the questionnaires, except for the self-reported questionnaire.

\section{Agitated Behavior in Dementia scale}

The Agitated Behavior in Dementia (ABID) includes items identified by Logsdon et $\mathrm{al}^{24}$ as being the most problematic in 
individuals with dementia and can be observed and described objectively. The caregivers first rated each behavior according to the frequency of occurrence during each of the 2 weeks immediately before the assessment on a scale of 0 to $3(0$, did not occur during the week; 1 , occurred 1-2 times during the week; 2, occurred 3-6 times during the week; 3, occurred daily or more often). The two weekly scores for each item were then added together, and the resulting item scores ranged from 0 to 6 . The item scores were summed to obtain a total score, with possible scores ranging from 0 to 96 . The caregivers then rated their own reactions to each problem behavior on a scale of 0 to 4 ( 0 , not upset; 4 , extremely upset). The caregiver's reactions were rated once for each item and then summed. The total reaction scores had a possible range of 0 to 64 . The reliability and the validity of the Japanese version of the ABID have been confirmed..$^{25}$ Three factors underlying the agitated behavior as evaluated using the Japanese version of the ABID were identified, ie, physically agitated behavior, verbally agitated behavior, and psychotic symptoms.

\section{Cohen-Mansfield Agitation Inventory}

The Cohen-Mansfield Agitation Inventory (CMAI) was originally developed to measure agitation in nursing home residents. ${ }^{26}$ The test consists of 29 observable agitated behaviors rated using a seven-point Likert-type scale according to the frequency of occurrence during the previous 2 weeks ( 0 , never occurred; 7 , occurred several times per hour). The reliability and validity of the CMAI have been established. ${ }^{26}$ A revised and expanded version of the CMAI, including 36 items, has been developed as an instrument to assess agitation among persons living in the community and has been used for senior day center participants. ${ }^{27}$ Homma et $\mathrm{al}^{28}$ confirmed the reliability and validity of the original 29 items of the CMAI in nursing home residents.

\section{Zarit Burden Interview}

The Zarit Burden Interview (ZBI) consists of 22 items rated using a five-point Likert scale (never, 0; nearly always, 4) with the aim of assessing caregiver burden..$^{29}$ The total burden is obtained by adding the scores for all items, with a range of 0 to 88 , and higher scores indicating a greater burden. The reliability and the validity of the Japanese version of this test battery have been confirmed. ${ }^{2}$

\section{Mini-Mental State Examination}

The Mini-Mental State Examination ${ }^{23}$ is a 30-point cognitive screening measure that is widely used to assess the severity of Alzheimer's disease. A lower score indicates greater impairment of cognitive function.

\section{Treatment}

Individual treatment intervention led by an experienced psychiatrist was performed based on the guidelines for Managing and Understanding Behavior Problems in Alzheimer Disease and Related Disorders. ${ }^{30}$ The treatment consisted of 12 sessions, each lasting 90 minutes once a week. Behavior management combines caregiver education and training in specific behavior techniques. ${ }^{31}$ The behavioral management program consisted of the following: sessions 1 and 2, which included psychoeducation regarding the nature of behavior problems in patients with dementia, and sessions 3 and 4, which began with the teaching of the ABCs of behavior change. ${ }^{30}$ In this approach, caregivers learn that " $\mathrm{A}$ " is the antecedent or triggering event that precedes the problem behavior, " $\mathrm{B}$ " is the behavior of concern, and " $C$ " is the consequence of that behavior. The behavior management intervention was started following the instructions regarding the ABCs. First, caregivers were instructed to gather information about the circumstances surrounding problems and to identify these problems according to the $\mathrm{ABC}$ approach. Caregivers were then asked to complete a weekly diary to monitor behavior problems. The therapist then checked the diary and instructed the caregiver with regard to how he or she might improve his or her skills so as to reduce the behavior problems identified. The remaining sessions focused on developing strategies to cope with behavior problems in patients with dementia according to the ABC approach. Caregivers were given homework tasks to apply the $\mathrm{ABC}$ approach by themselves. During these sessions, both the therapist and the caregivers reviewed the homework, and the therapists provided feedback to modify and develop the skills of the caregivers. Finally, the caregivers were instructed to discuss their issues and to make treatment plans for self-management in the future.

\section{Statistical analysis}

We used the Statistical Package for the Social Sciences (Windows version 17.0) (SPSS Inc, Chicago, IL, USA) for the statistical analyses. All the statistical tests were two-tailed, and a $P$ value $<0.05$ was regarded as being statistically significant for changes in clinical treatment effects before and after the intervention. Because our sample size was relatively small, we used nonparametric tests and the Wilcoxon's signed rank test. This study was conducted as exploratory research. Based on several similar previous studies, ${ }^{7,9}$ we estimated that a sample size of about 18 participants was needed. 


\section{Results}

\section{Demographic and clinical characteristics}

Baseline demographic data for the patients with dementia are summarized in Table 1. Of the 22 patients with dementia (patients with Alzheimer's disease, $n=14$; patients with dementia with Lewy bodies, $\mathrm{n}=8$ ) who participated in the intervention study, four patients suffering from dementia with Lewy bodies did not complete the study because they were transferred to another hospital. Among the remaining 18 patients, four with Alzheimer's disease were taking risperidone $0.5 \mathrm{mg} / \mathrm{day}$, and this dose was maintained throughout the intervention. The risperidone had been first prescribed approximately 6 months before the start of the presently reported intervention.

\section{Clinical findings before and after treatment} As shown in Table 2, mean total CMAI and ABID scores decreased dramatically after 12 weeks of behavioral management $(P<0.001)$. Furthermore, the mean ZBI score also decreased significantly after behavioral management. Physically and verbally agitated behavior scores improved significantly on the ABID, although symptoms of psychosis did not improve after the intervention. Further, the mean Mini-Mental State Examination score did not change significantly after the intervention. Significant differences in clinical outcomes after treatment were not observed between male and female patients with dementia.

\section{Discussion}

In the present pilot study, we examined the effectiveness of behavioral management with regard to agitated behaviors in patients with dementia. As far as we know, this is the first study to examine the use of behavioral management techniques in Japan. Significant reductions in agitated behavior were observed in patients with dementia when evaluated using

Table I Demographic and clinical characteristics of dementia patients and caregivers

\begin{tabular}{lll}
\hline & \multicolumn{2}{l}{ Mean \pm SD $(\mathbf{n}=18)$} \\
\cline { 2 - 3 } & Patients & Caregivers \\
\hline Type of dementia & 14 & - \\
AD & 4 & - \\
DLB & $7 / 11$ & $11 / 7$ \\
Male/female & $71 \pm 5.7$ & $70 \pm 6.1$ \\
Age, years & $9.5 \pm 0.5$ & $9.6 \pm 0.5$ \\
Education, years & $4.1 \pm 1.2$ & - \\
Duration of illness, years &
\end{tabular}

Note: Data are presented as the mean $\pm S D$.

Abbreviations: AD, Alzheimer's disease; DLB, dementia with Lewy bodies.
Table 2 Clinical findings before and after behavior management approach in dementia patients

\begin{tabular}{lccl}
\hline & \multicolumn{2}{c}{ Mean \pm SD } & $P$ \\
\cline { 2 - 3 } & Baseline & $\begin{array}{l}\text { After } \\
\text { intervention }\end{array}$ & \\
\hline MMSE & $15.2 \pm 3.6$ & $14.8 \pm 3.5$ & 0.166 \\
ZBI & $44.1 \pm 19.9$ & $36.3 \pm 16.9$ & $<0.00$ I \\
CMAI & $39.1 \pm 27.1$ & $32.3 \pm 25.2$ & 0.003 \\
ABID & & & \\
Frequency questions & $51.1 \pm 12.6$ & $43.7 \pm 11.4$ & $<0.00$ I \\
$\quad$ Physical agitated behavior & $14.5 \pm 10.3$ & $11.5 \pm 8.4$ & 0.001 \\
$\quad$ Verbally agitated behavior & $28.7 \pm 7.6$ & $24.6 \pm 6.3$ & $<0.00$ I \\
$\quad$ Psychosis symptoms & $7.8 \pm 3.6$ & $7.5 \pm 4.1$ & 0.366 \\
Reaction ratings & $54.9 \pm 28.4$ & $43.3 \pm 24.3$ & $<0.00$ I \\
$\quad$ Physical agitated behavior & $17.6 \pm 14.4$ & $13.5 \pm 11.7$ & 0.001 \\
$\quad$ Verbally agitated behavior & $32.6 \pm 16.1$ & $25.2 \pm 12.9$ & $<0.00$ I \\
Psychosis symptoms & $4.6 \pm 3.5$ & $4.5 \pm 3.6$ & 0.748 \\
\hline
\end{tabular}

Notes: Data are presented as the mean \pm SD. Except for the MMSE score and the psychosis symptoms of the ABID, significant differences before and after intervention were observed for all the items.

Abbreviations: MMSE, Mini-mental state examination; ZBI, Zarit Burden Interview; CMAI, The Cohen-Mansfield Agitation Inventory; ABID, Agitated Behavior in Dementia scale.

both the ABID and the CMAI. Furthermore, large reductions in the care burden of caregivers, as evaluated using the ZBI, were also observed. Thus, our study provides additional support for the effectiveness of nonpharmacological interventions for agitated behaviors in patients with dementia.

However, the present study had several limitations. First, although the physically and verbally agitated behavior scores improved on the ABID, our study failed to show any improvement in symptoms of psychosis according to this scale. Psychotic symptoms are well known to be distinct from agitation in patients with dementia. ${ }^{32}$ Delusions in dementia can be categorized into several types, including persecutory delusions and misidentification delusions associated with false beliefs. These delusions are regarded as being associated with various types of cognitive dysfunction, but mainly executive dysfunction. ${ }^{33}$ Changes in behavior are difficult to achieve in patients with dementia suffering from both delusions and hallucinations.

Second, although all patients with Alzheimer's disease completed the study, half of the patients suffering from dementia with Lewy bodies $(n=4)$ did not. Dementia with Lewy bodies is characterized by recurrent visual hallucinations, fluctuations in attention, and rapid eye movement sleep disorders. Neuropsychiatric symptoms are more severe and prevalent in patients who have dementia with Lewy bodies than in those with Alzheimer's disease. ${ }^{1}$ Thus, these severe symptoms are major sources of difficulty in identifying behavior problems according to the $\mathrm{ABC}$ approach. 
Third, our study did not include placebo control group. We relied on within-group changes to examine the effects of behavioral management techniques. In addition, the sample size was relatively small. Thus, our results may overestimate the effect of behavioral management. A large-scale, randomized clinical trial is needed to clarify the effect of behavioral management on agitated behavior in patients with dementia.

Fourth, whether the effects of behavioral management techniques persisted after the end of the study is unclear. Weiner et $\mathrm{al}^{34}$ conducted a 12-month follow-up study of a 4-month randomized clinical trial and failed to show any long-term effects of behavioral management.

Fifth, Cohen-Mansfield ${ }^{35}$ proposed that agitated behaviors could be divided into two dimensions, ie, aggressive versus nonaggressive, and verbal versus physical behaviors. ${ }^{35}$ Several items (eg, "harmful to self") that were included in the physically agitated behavior item in the ABID corresponded to the agitated behaviors that Cohen-Mansfield ${ }^{35}$ described as being physically aggressive. However, in an examination of the factor structure of the ABID in our previous study, ${ }^{25}$ agitated behaviors were clearly not divided into two dimensions (aggressive versus nonaggressive). Unlike the CMAI, the ABID may not have the sensitivity to discriminate between physically aggressive and nonaggressive behaviors.

Sixth, the main strategy of this study was to provide a basis for consequent strategies. However, in terms of geriatric psychiatry, an antecedent control, such as alteration of the environment to preclude behavioral problems, is regarded as a clinically more useful approach. ${ }^{20,36} \mathrm{An}$ antecedent control is an appropriate approach for patients with dementia, requiring less input from caregivers in terms of effort and time. Thus, in future studies, we plan to develop more adaptive antecedent controls for agitated behavior in patients with dementia aimed at providing more benefits relative to this study.

Despite these limitations, behavioral management is easy and safe to perform, and is likely to be beneficial to caregivers suffering from distress because of agitated behaviors. In the future, a well designed study to develop more fully the potential of behavioral management techniques is needed for a variety of environments, including nursing homes.

\section{Acknowledgment}

The authors gratefully acknowledge a Grant-in-Aid for Scientific Research (22530750, 22591293) from the Ministry of Education, Culture, Sports, Sciences, and Technology in Japan.

\section{Disclosure}

The authors report no conflicts of interest in this work.

\section{References}

1. Hirono N, Mori E, Tanimukai S, et al. Distinctive neurobehavioral features among neurodegenerative dementias. $J$ Neuropsychiatry Clin Neurosci. 1999;11(4):498-503.

2. Hirono N, Kobayashi H, Mori E. Caregiver burden in dementia: evaluation with a Japanese version of the Zarit caregiver burden interview. No to Shinkei =Brain and Nerve. 1998;50(6):561-567.

3. Cohen-Mansfield J, Billig N. Agitated behaviors in the elderly. I. A conceptual review. J Am Geriatr Soc. 1986;34(10):711-721.

4. Sultzer DL, Davis SM, Tariot PN, et al. Clinical symptom responses to atypical antipsychotic medications in Alzheimer's disease: phase 1 outcomes from the CATIE-AD effectiveness trial. Am J Psychiatry. 2008;165(7):844-854.

5. Schneider LS, Dagerman KS, Insel P. Risk of death with atypical antipsychotic drug treatment for dementia: meta-analysis of randomized placebo-controlled trials. JAMA. 2005;294(15):1934-1943.

6. Kales HC, Valenstein M, Kim HM, et al. Mortality risk in patients with dementia treated with antipsychotics versus other psychiatric medications. Am J Psychiatry. 2007;164(10):1568-1576.

7. Teri L, Logsdon RG, Peskind E, et al. Alzheimer's Disease Cooperative Study. Treatment of agitation in AD: a randomized, placebo-controlled clinical trial. Neurology. 2000;55(9):1271-1278.

8. Teri L, Gibbons LE, McCurry SM, et al. Exercise plus behavioral management in patients with Alzheimer disease: a randomized controlled trial. JAMA. 2003;290(15):2015-2022.

9. McCurry SM, Gibbons LE, Logsdon RG, Vitiello MV, Teri L. Nighttime insomnia treatment and education for Alzheimer's disease: a randomized, controlled trial. J Am Geriatr Soc. 2005;53(5):793-802.

10. Livingston G, Johnston K, Katona C, Paton J, Lyketsos CG. Systematic review of psychological approaches to the management of neuropsychiatric symptoms of dementia. Am J Psychiatry. 2005;162(11): 1996-2021.

11. Ayalon L, Gum AM, Feliciano L, Arean PA. Effectiveness of nonpharmacological interventions for the management of neuropsychiatric symptoms in patients with dementia: a systematic review. Arch Intern Med. 2006;166(20):2182-2188.

12. Logsdon RG, McCurry SM, Teri L. Evidence-based psychological treatments for disruptive behaviors in individuals with dementia. Psychol Aging. 2007;22(1):28-36.

13. Beck CK, Vogelpohl TS, Rasin JH, et al. Effects of behavioral interventions on disruptive behavior and affect in demented nursing home residents. Nurs Res. 2002;51(4):219-228.

14. Suhr J, Anderson S, Tranel D. Progressive muscle relaxaion in the management of behvioral disturbance in Alzheimer's disease. Neuropsychol Rehabil. 1999;9:31-44.

15. Cooper C, Mukadam N, Katona C, et al. Systematic review of the effectiveness of non-pharmacological interventions to improve quality of life of people with dementia. Int Psychogeriatr. 2012;24(6):856-870.

16. McCallion P, Toseland RW, Freeman K. An evaluation of a family visit education program. J Am Geriatr Soc. 1999;47(2):203-214.

17. Gitlin LN, Winter L, Dennis MP, Hodgson N, Hauck WW. A biobehavioral home-based intervention and the well-being of patients with dementia and their caregivers: the COPE randomized trial. JAMA. 2010;304(9):983-991.

18. Proctor R, Burns A, Powell HS, et al. Behavioural management in nursing and residential homes: a randomised controlled trial. Lancet. 1999;354(9172):26-29.

19. Teri L, McCurry SM, Logsdon R, Gibbons LE. Training community consultants to help family members improve dementia care: a randomized controlled trial. Gerontologist. 2005;45(6):802-811.

20. Narumoto J, Miya H, Shibata K, et al. Challenging behavior of patients with frontal dysfunction managed successfully by behavioral intervention. Psychogeriatrics. 2009;9:147-150. 
21. McKhann G, Drachman D, Folstein M, Katzman R, Price D, Stadlan EM. Clinical diagnosis of Alzheimer's disease: report of the NINCDS-ADRDA Work Group under the auspices of Department of Health and Human Services Task Force on Alzheimer's Disease. Neurology. 1984;34(7):939-944.

22. McKeith IG, Galasko D, Kosaka K, et al. Consensus guidelines for the clinical and pathologic diagnosis of dementia with Lewy bodies (DLB): report of the consortium on DLB international workshop. Neurology. 1996;47(5):1113-1124.

23. Folstein MF, Folstein SE, McHugh PR. "Mini-mental state". A practical method for grading the cognitive state of patients for the clinician. J Psychiatr Res. 1975;12(3):189-198.

24. Logsdon RG, Teri L, Weiner MF, et al. Assessment of agitation in Alzheimer's disease: the agitated behavior in dementia scale. Alzheimer's Disease Cooperative Study. J Am Geriatr Soc. 1999;47(11): 1354-1358.

25. Torii K, Nakaaki S, Banno K, et al. Reliability and validity of the Japanese version of the Agitated Behaviour in Dementia Scale in Alzheimer's disease: three dimensions of agitated behaviour in dementia. Psychogeriatrics. 2011;11(4):212-220.

26. Cohen-Mansfield J, Marx MS, Rosenthal AS. A description of agitation in a nursing home. J Gerontol. 1989;44(3):77-84.

27. Cohen-Mansfield J, Werner P, Watson V, Pasis S. Agitation among elderly persons at adult day-care centers: the experiences of relatives and staff members. Int Psychogeriatr. 1995;7(3):447-458.

28. Homma A, Niina R, Ishii T, et al. Assessment of validity of a Japanese version of Cohen-Mansfield Agitation Inventory. Jpn J Geriatr Psychiatry. 2002;13(7):831-835.
29. Zarit SH, Reever KE, Bach-Peterson J. Relatives of the impaired elderly: correlates of feelings of burden. Gerontologist. 1980;20(6):649-655.

30. Teri L. Management and understaing behavior problems in Alzheimer's disease and related disorders. Videotape training program. Seattle, WA: University of Washington; 1990.

31. Teri L, Logsdon RG. Assessment and management of behavioral disturbances in Alzheimer disease. Compr Ther. 2000;26(3): 169-175.

32. Aalten P, de Vugt ME, Lousberg R, et al. Behavioral problems in dementia: a factor analysis of the neuropsychiatric inventory. Dement Geriatr Cogn Disord. 2003;15(2):99-105.

33. Holt AE, Albert ML. Cognitive neuroscience of delusions in aging. Neuropsychiatr Dis Treat. 2006;2(2):181-189.

34. Weiner MF, Tractenberg RE, Sano M, et al. No long-term effect of behavioral treatment on psychotropic drug use for agitation in Alzheimer's disease patients. J Geriatr Psychiatry Neurol. 2002;15(2): 95-98.

35. Cohen-Mansfield J. Agitation in the elderly definitional and theoretical conceptualizations. In: Hay DP, Klein DT, Hay LK, Grossberg GT, Kennedy JS, editors. Agitation in Patients with Dementia: A Practical Guide to Diagnosis and Management. Washington, DC: American Psychiatric Publishing Inc; 2003.

36. Molinari V, Edelstein BA. Commentary on the current status and the future of behavior therapy in long-term care settings. Behav Ther. 2011; 42(1):59-65.
Neuropsychiatric Disease and Treatment

\section{Publish your work in this journal}

Neuropsychiatric Disease and Treatment is an international, peerreviewed journal of clinical therapeutics and pharmacology focusing on concise rapid reporting of clinical or pre-clinical studies on a range of neuropsychiatric and neurological disorders. This journal is indexed on PubMed Central, the 'PsycINFO' database and CAS.

\section{Dovepress}

The manuscript management system is completely online and includes a very quick and fair peer-review system, which is all easy to use. Visit http://www.dovepress.com/testimonials.php to read real quotes from published authors. 